\title{
Predictive Factors And Long-Term Visual Outcomes After Anti-Vascular Endothelial Growth Factor Treatment Of Retinal Angiomatous Proliferation
}

This article was published in the following Dove Press journal: Clinical Ophthalmology

\section{Maiko Maruyama-Inoue Shimpei Sato (iD) Shin Yamane Kazuaki Kadonosono}

Department of Ophthalmology, Yokohama City University Medical Center, Yokohama, Japan
Correspondence: Maiko Maruyama-Inoue Department of Ophthalmology, Yokohama City University Medical Center, 4-57 Urafune-cho, Minami-ku, Yokohama, Kanagawa 232-0024, Japan Tel +8I $4526 \mid 5656$

$\mathrm{Fax}+81452538490$

Email maicoo@urahp.yokohama-cu.ac.jp
Purpose: To report the results of 9-year follow-up examinations and predictive factors for visual acuity outcome after intravitreal injection of anti-vascular endothelial growth factor (VEGF) agents to treat retinal angiomatous proliferation (RAP).

Methods: We conducted a retrospective observational study of 85 treatment-naïve eyes in 61 patients ( 21 men, 40 women; age range, 70-95 years; mean age, 84.0 years) treated with intravitreal injections of anti-VEGF agents. All patients received three consecutive monthly injections as an induction treatment. During the maintenance phase, the patients received intravitreal injections as needed or fixed dosing. The primary outcome measures were bestcorrected visual acuity (BCVA) during the follow-up period. Furthermore, we investigated potential predictive factors of improvement in visual acuity. The proportion of patients who developed specific complications were also analyzed.

Results: The mean BCVA gradually decreased from 0.58 at baseline to 0.70 at 36 months $(P=0.146), 0.82$ at 48 months $(P=0.004)$, and 0.92 at 108 months $(P=0.021)$. Improvement in visual acuity at the final visits was associated with baseline visual acuity and central foveal thickness. Massive subretinal hemorrhage, fibrotic scars, and macular atrophy developed in 4 (4.7\%), 9 (10.6\%), and 50 (56.8\%) eyes, respectively, at the final visits, and were all significantly associated with final visual acuity $(P=0.013, P<0.001$, and $P=0.001$, respectively).

Conclusion: Long-term stabilization of vision in patients with RAP, regardless of treatment modality, was difficult to achieve by using intravitreal injections of anti-VEGF agents. Earlier detection and treatment are important to maintain visual acuity in patients with RAP. Keywords: ranibizumab, aflibercept, retinal angiomatous proliferation, age-related macular degeneration, intravitreal injection

\section{Introduction}

Yannuzzi et al first introduced the term retinal angiomatous proliferation (RAP) as a variant of neovascular age-related macular degeneration (AMD), which originates from the retina and extends into the subretinal space with or without pigment epithelial detachment (PED). ${ }^{1}$ The clinical course of RAP differs from that of typical exudative AMD, with unfavorable visual outcomes. ${ }^{2}$ Several methods of treatments for RAP, such as laser photocoagulation, ${ }^{3,4}$ photodynamic therapy, ${ }^{5,6}$ surgical ablation, ${ }^{7,8}$ and transpupillary thermotherapy, ${ }^{3,9}$ have been studied, but none have been found to be completely successful because of difficulty completing obliteration of the lesions, progressive scarring, and recurrent exudation. 
Recently, anti-vascular endothelial growth factor (VEGF) agents, such as ranibizumab (Lucentis ${ }^{\circledR}$; Novartis Pharma AG, Basel, Switzerland, and Genentech, South San Francisco, CA, USA) and aflibercept (Eylea ${ }^{\circledR}$; Bayer HealthCare, Berlin, Germany), have been used extensively for the treatment of AMD. The use of these agents to treat RAP has also achieved better visual outcomes. ${ }^{10-12}$ Previously, our group showed that intravitreal ranibizumab administered on an as-needed basis, which is known as "pro re nata" (PRN), after 3 initial monthly doses were effective for maintaining visual acuity, as evaluated at a 3 -year follow-up examination. ${ }^{13}$ However, no predictive factors for visual outcome have been investigated over the long term.

Furthermore, it is known that many complications can limit visual improvement after anti-VEGF treatment. Several reports have concluded that enlargement of geographic atrophy (GA) occurred frequently in patients with RAP after treatment, ${ }^{14,15}$ which might result in severe visual loss. Another study reported that the incidence of subretinal hemorrhage (SRH) increased steadily in patients with RAP and was $>10 \%$ after the 5 -year follow-up period. ${ }^{16}$

The purpose of the present study was to analyze the long-term functional results of anti-VEGF agents for the treatment of RAP. We also evaluated factors that could potentially influence final visual acuity. Furthermore, the proportion of patients who developed specific complications were also analyzed.

\section{Patients And Method}

We conducted a retrospective observational study of 85 eyes in 61 consecutive Japanese patients with RAP who were followed for $\geq 12$ months after the first intravitreal administration of ranibizumab or aflibercept. All patients were treated at the Yokohama City University Medical Center between April 2009 and March 2017. The study was conducted according to the principles of the Declaration of Helsinki, and consent statement was both written and informed from all eligible patients. The ethics committee of the Yokohama City University Medical Center approved the study protocol.

The inclusion criteria were the presence of RAP as diagnosed on the basis of clinical and angiographic findings, treatment-naïve, availability for follow-up $\geq 12$ months after the first intravitreal administration of antiVEGF agents, and a best-corrected visual acuity (BCVA) of $\geq 20 / 400$ at baseline. Patients who had previously received treatment for RAP, such as laser photocoagulation, submacular surgery, photodynamic therapy, or intravitreal injection of any other anti-VEGF agent, were excluded from the study. Furthermore, patients with eye diseases that could potentially influence the visual acuity of the studied eye, such as glaucoma, macular hole, diabetic retinopathy, or rhegmatogenous retinal detachment (RRD), were also excluded.

BCVA measurements, spectral-domain optical coherence tomography (SD-OCT), color fundus photography, fluorescein angiography, indocyanine green angiography, and fundus autofluorescence (FAF) imaging were performed in all patients at baseline. At each follow-up visit, measurements of BCVA and SD-OCT imaging were performed. Fundus photography and FAF were performed when necessary. The BCVA value was converted to the logarithm of the minimum angle of resolution $(\log M A R)$ for statistical analysis. The presence/absence of reticular pseudodrusen (RPD) was investigated by using SD-OCT and color fundus photography.

All patients received 3 consecutive monthly intravitreal injections of $0.5 \mathrm{mg} / 0.05 \mathrm{~mL}$ of ranibizumab or $2 \mathrm{mg} / 0.05$ $\mathrm{mL}$ of aflibercept through the pars plana via a 30-gage needle as an induction treatment. For patients treated with PRN, the intravitreal injections were repeated during the maintenance phase if any of the following changes were observed by the evaluating physician: 1) a visual acuity loss of $0.2 \log$ MAR vision or more, 2) new macular hemorrhage, or 3) evidence of persistent or recurrent subretinal fluid accumulation, intraretinal edema, or enlargement of the PED as diagnosed by using SD-OCT at the 1- or 2-month follow-up examination, as judged by the retina specialists (M.M. or S.S.). Patients with fixed dosing were first instructed to receive monthly follow-up examinations. Then, based on the intervals of recurrent disease, fixed treatment intervals were decided to avoid the burden of frequent monitoring.

The primary outcome measure was change in the BCVA from preinjection to postinjection at 108 months after initial treatment by using a Student's $t$-test. The secondary outcome was to investigate predictive factors influencing the improvement of visual acuity and the final visual acuity. Explanation variables, such as age, sex, visual acuity at baseline, anti-VEGF agents (ranibizumab or aflibercept), number of injections, greatest linear dimension (GLD) at baseline, central foveal thickness (CFT) at baseline, central choroidal thickness (CCT) at baseline, and the presence/ absence of RPD at baseline, were included in a multiple regression analysis by stepwise selection. The response variables were the improvement in visual acuity, which was defined as the difference between pre- and postinjection 
BCVA at final visits, and the final visual acuity. These visual acuity parameters were also compared between the PRN group and the fixed dosing group using the $t$-test. Furthermore, the proportion of patients who developed a massive SRH, fibrotic scars, and macular atrophy was analyzed. Massive SRH was defined by a thick submacular and/or subretinal pigment epithelium (RPE) hemorrhages that extend past the equator in $\geq 2$ quadrants. Scars were classified as fibrotic with well-demarcated elevated mounds of yellowish white tissue. Macular atrophy was defined as loss of RPE and outer retina at the foveal center, evaluated by color fundus photography, SD-OCT, and FAF. The association between these complications and the final visual acuity was analyzed by using the unpaired $t$-test. Additionally, the associations between the development of macular atrophy and the patient characteristics, including age, sex, baseline visual acuity, CFT, CCT, GLD, presence/ absence of RPD, anti-VEGF agents, and the number of injections, were investigated by using multiple logistic regression analysis.

All statistical analyses were performed by using EkuseruToukei 2012 (Social Survey Research Information, Tokyo, Japan). $P$-values $<0.05$ were considered as an indication of statistical significance.

\section{Results}

A total of 85 eyes were included in this study. The mean follow-up period was $45.7 \pm 26.4$ months. The baseline characteristics and clinical data for each patient are shown in Table 1. Of the 85 eyes included in this study, 68 patients were treated with PRN and 17 patients with fixed dosing. Of the 61 patients included in the series, 21 were men and 40 were women. The patient age ranged from 70 to 95 years (mean age, $84.0 \pm 6.7$ years). At baseline, 8 (9.4\%) eyes had a stage 1 lesion, 25 (29.4\%) eyes had a stage 2 lesion without a PED, 28 (33.0\%) eyes had stage 2 lesions with a PED, and the remaining 24 (28.2\%) eyes had stage 3 lesions.

Overall, the mean $\log$ MAR visual acuity at baseline was $0.58 \pm 0.42$. The mean BCVA gradually decreased from 0.58 at baseline to 0.70 at 36 months ( $P=0.146$ ), to 0.82 at 48 months $(P=0.004)$, and to 0.92 at 108 months $(P=0.021)$. The postinjection BCVA values at 48, 60, 72, 84, 96, and 108 months were significantly worsened, than the value at baseline $(P=0.004, P=0.029, P=0.002, P=0.004, P=0.011$, and $P=0.021$, respectively). The mean numbers of injections were 4.6 for the first year and 2.3-3.6 injections per year from years 2 to 9 (Figure 1).

Twelve (14.1\%) of the 85 eyes had an improvement in visual acuity of $\geq 0.3 \log$ MAR at the final visit. The visual acuity of $41(48.2 \%)$ eyes remained unchanged, and the remaining $32(37.7 \%)$ eyes exhibited deterioration in visual acuity of $\geq 0.3 \log$ MAR.

Table 2 shows the results of the stepwise multiple regression analyses. Better baseline visual acuity was associated with better visual acuity at final visits and a lesser

Table I Clinical Characteristics Of The Patients With RAP

\begin{tabular}{|l|l|}
\hline Number of patients & $6 \mathrm{I}$ \\
\hline Number of eyes & 85 \\
\hline Age, mean \pm SD, year & $84.0 \pm 6.7$ \\
\hline Sex (male/female) & $21 / 40$ \\
\hline Baseline logMAR visual acuity, mean \pm SD & $0.58 \pm 0.42$ (Snellen equivalent 20/76) \\
\hline RAP stage (I/2/3) & $8 / 53 / 24$ \\
\hline Presence of RPD, present/none (\%) & $40 / 45(47 / 53)$ \\
\hline Central foveal thickness, mean \pm SD $(\mu \mathrm{m})$ & $547 \pm 287$ \\
\hline Central choroidal thickness, mean \pm SD $(\mu \mathrm{m})$ & $133 \pm 60$ \\
\hline Greatest linear dimension, mean \pm SD $(\mu \mathrm{m})$ & $3034 \pm 1288$ \\
\hline Anti-VEGF agents (ranibizumab/aflibercept)(\%) & $69 / 16(81 / 19)$ \\
\hline Treatment modality (PRN/fixed dosing) & $68 / 17(80 / 20)$ \\
\hline
\end{tabular}

Abbreviations: RAP, retinal angiomatous proliferation; SD, standard deviation; logMAR, logarithm of the minimum angle of resolution; RPD, reticular pseudodrusen; VEGF, vascular endothelial growth factor. 


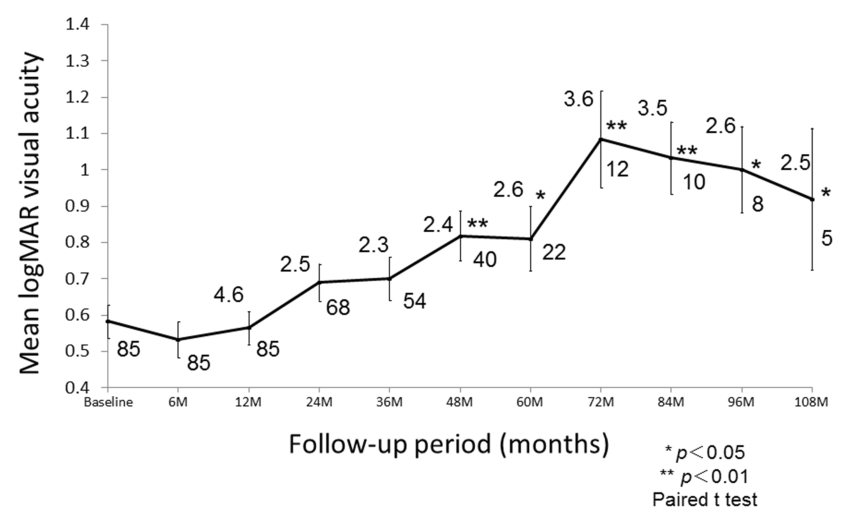

Figure I Graph showing the changes in the mean values of the logMAR BCVA from baseline until 108 months after the first anti-VEGF injection. The difference in postinjection BCVA relative to the baseline level was maintained throughout the 36-month period but was significantly decreased from 48 months to 108 months. The upper numbers show the number of injections. The lower numbers show the number of patients who were able to be examined during the follow-up.

degree of visual improvement $(P=0.003$ and $P<0.001$, respectively). A smaller CFT was also associated with better visual acuity at final visits and a greater degree of visual improvement ( $P=0.015$ for both comparisons). On the other hand, there were no significant associations between any of the other explanation variables used in this study (age, sex, anti-VEGF agents, number of injections, GLD, CCT, and presence or absence of RPD) and the postoperative visual acuity parameters (all $P>0.05$ ). Regarding treatment modality, there were no significant differences of final visual acuity and visual improvement between the PRN group and the fixed dosing group ( $P=0.586$ and $P=0.689$, respectively).

During the follow-up periods, massive SRH developed in $4(4.7 \%)$ eyes, and fibrotic scar occurred in $9(10.6 \%)$ eyes. On the other hand, macular atrophy developed in 50 $(56.8 \%)$ eyes at the final visits. Statistical analysis showed that final visual acuity was significantly influenced by the incidence of massive SRH, presence/absence of fibrotic scar, and presence/absence of macular atrophy $(P=0.013$, $P<0.001$, and $P=0.001$, respectively) (Figure 2).

Furthermore, Table 3 shows the prognostic factors for macular atrophy. Multiple logistic regression analysis showed that baseline BCVA, age, and number of injections were significantly associated with macular atrophy at the final visits $(P=0.049, P=0.049$, and $P=0.014$, respectively). On the other hand, there were no significant associations between any of the other variables used in this study (sex, GLD, CFT, CCT, and presence/absence of $\mathrm{RPD}$, and anti-VEGF agents) and the presence of macular atrophy $(P>0.05)$.

No systemic or ocular side effects, such as endophthalmitis and RRD, were noted in any of the patients during the follow-up period. Figures 3, 4, and 5 show case reports of representative patients with RAP.

\section{Discussion}

Our study demonstrated that it was difficult to stabilize vision in patients with RAP by administering intravitreal injections of anti-VEGF agents over the long term regardless of the treatment modality. Baseline BCVA and CFT were significantly associated with final BCVA and improvement in BCVA. This is the first report to describe the treatment response to anti-VEGF in patients with RAP for $>9$ years. Furthermore, not only development of macular atrophy but also occurrence of fibrotic scarring and massive SRH could result in deterioration of visual acuity in patients with RAP.

Several studies have reported the efficacy of intravitreal ranibizumab or aflibercept for the treatment of RAP, although GA develops for prolonged periods of time. ${ }^{12,17}$ Our group also previously reported that anti-VEGF monotherapy was effective for maintaining or improving the visual acuity of patients with RAP at 3 years after the

Table 2 Stepwise Multiple Regression Analysis Of The BCVA At Final Visit And Improvement Of The Visual Acuity

\begin{tabular}{|c|c|c|c|c|c|c|}
\hline \multirow{3}{*}{$\begin{array}{l}\text { Explanation } \\
\text { Variables }\end{array}$} & \multicolumn{6}{|l|}{ Response Variables } \\
\hline & \multicolumn{3}{|c|}{ Post-Injection BCVA* } & \multicolumn{3}{|c|}{ Improvement Of The Visual Acuity** } \\
\hline & $\begin{array}{l}\text { Partial Regression } \\
\text { Coefficient }\end{array}$ & $\begin{array}{l}\text { Standard } \\
\text { Error }\end{array}$ & p-Value & $\begin{array}{l}\text { Partial Regression } \\
\text { Coefficient }\end{array}$ & $\begin{array}{l}\text { Standard } \\
\text { Error }\end{array}$ & p-Value \\
\hline Baseline visual acuity & 0.355 & 0.1149 & 0.003 & -0.645 & 0.1149 & $<0.001$ \\
\hline Central foveal thickness & 0.0004 & 0.0002 & 0.015 & 0.0004 & 0.0002 & 0.015 \\
\hline
\end{tabular}

Notes: *Post-injection BCVA = BCVA at final visit. **Improvement of the visual acuity $=$ the difference between baseline visual acuity and BCVA at final visit.

Abbreviations: BCVA, best-corrected visual acuity; RPD, reticular pseudodrusen; VEGF, vascular endothelial growth factor. 

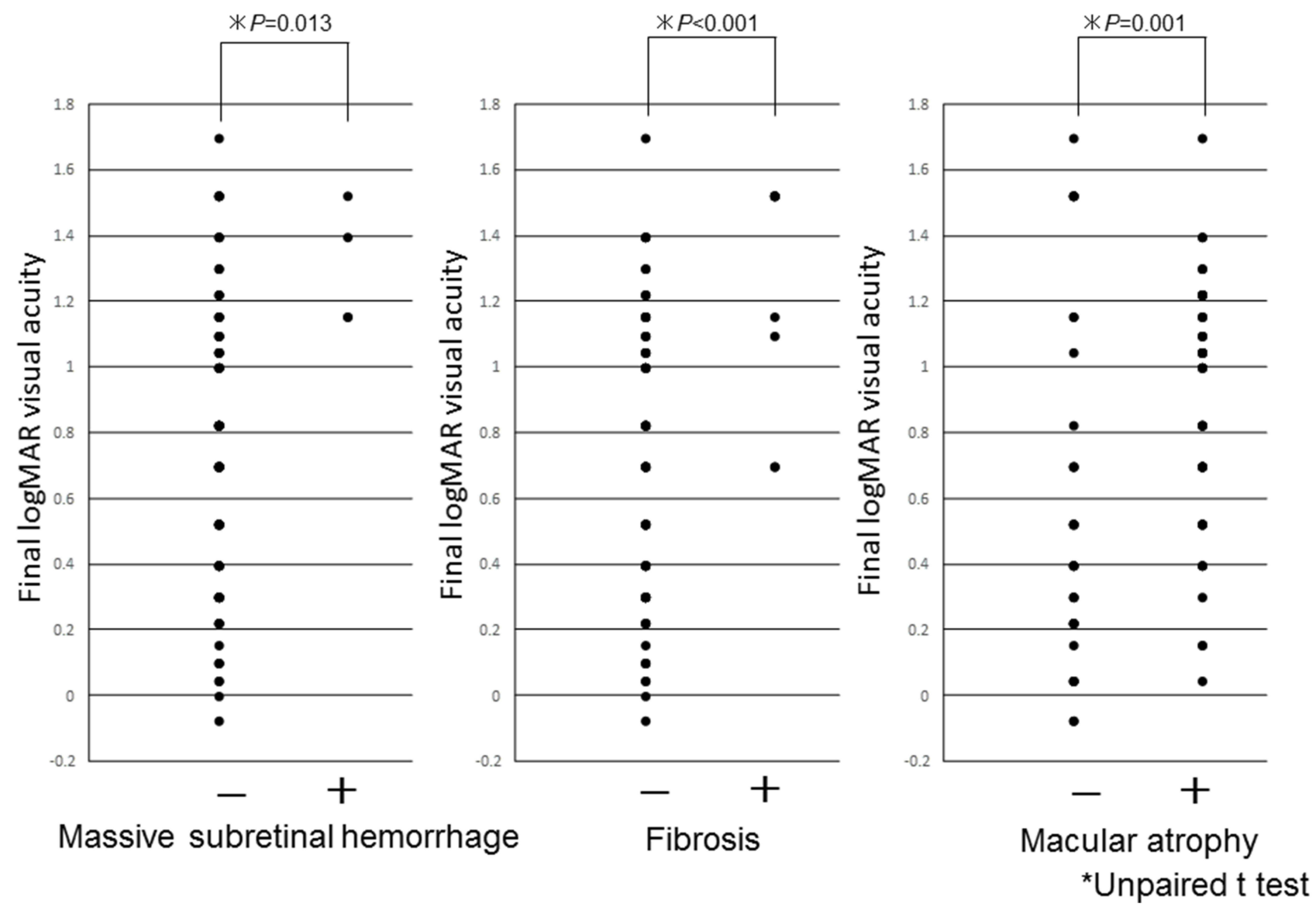

Figure 2 An association between complications and final visual acuity. Final BCVA was significantly influenced by the incidence of massive subretinal hemorrhage, presence/ absence of fibrotic scar, and presence/absence of macular atrophy $(P=0.013, P<0.00 \mathrm{I}$, and $P=0.00 \mathrm{I}$, respectively).

start of treatment. ${ }^{13}$ However, in this study, although visual acuity was maintained for 3 years, maintenance after 3 years was limited. As previous studies have shown, development of macular atrophy is one possible explanation for the decrease in visual acuity in patients with RAP. The CATT research group reported that $24 \%$ of AMD patients showed GA for the follow-up at 1 year ${ }^{17}$ and $36.8 \%$ at 3 years. ${ }^{18}$ In the SEVEN-UP study in patients with AMD, macular atrophy was present in $98 \%$

Table 3 Multiple Logistic Regression Analysis To Predict The Development Of Macular Atrophy

\begin{tabular}{|l|l|l|l|}
\hline $\begin{array}{l}\text { Independent } \\
\text { Variables }\end{array}$ & $\begin{array}{l}\text { Odds } \\
\text { Ratio }\end{array}$ & $\mathbf{9 5 \%} \mathbf{C l}$ & $\mathbf{p}$-Value \\
\hline Baseline visual acuity & 3.33 & $1.007-11.023$ & 0.049 \\
Age & 1.08 & $1.000-1.165$ & 0.049 \\
Number of injections & 1.07 & $1.015-1.138$ & 0.014 \\
\hline
\end{tabular}

Notes: Excluded variables: sex, central choroidal thickness, central choroidal thickness, greatest linear dimension, presence of RPD, and anti-VEGF agents. Abbreviations: $\mathrm{Cl}$, confidence interval; RPD, reticular pseudodrusen; VEGF, vascular endothelial growth factor. of the patients at a mean of 7.3 years. ${ }^{19}$ Baek et al reported that GA developed in $60 \%$ of the RAP patients, and the foveal center was involved in about one-fifth of the patients at the follow-up of 3 years. ${ }^{20}$ In this study, $58.8 \%$ of the patients showed macular atrophy at a mean follow-up of about 4 years, which was consistent with the findings of previous studies. Furthermore, fibrotic scars and massive SRH were also significant factors associated with visual deterioration, which was consistent with the findings of previous studies. ${ }^{16,21}$ Therefore, it is important to prevent these complications to maintain BCVA in patients with RAP. Another possibility for the decrease in visual acuity is that some patients may have been undertreated in this study. Although we administered injections on the basis of our definition to retreat, irreversible damage to the photoreceptors could have occurred because of continuous or recurrent exudative changes. Stricter injections may be needed to maintain BCVA over the long term, although they could also be a risk factor for progression of macular atrophy. 


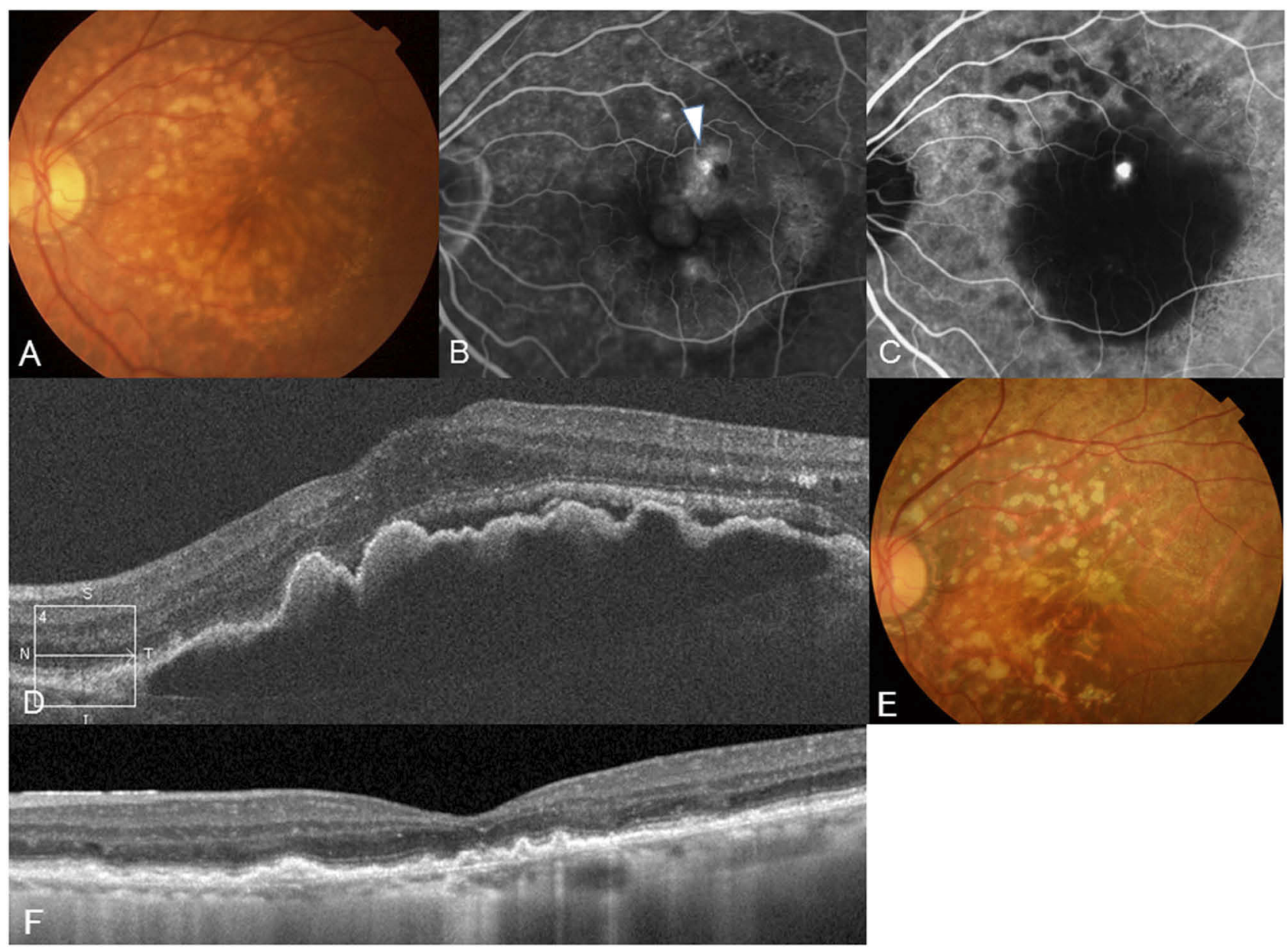

Figure 3 A 78-year-old woman presented with decreased visual acuity in her left eye. (A) A color fundus photograph of the left eye showing multiple soft drusen and reticular pseudodrusen. (B) Late-phase fluorescein angiographic image of the fundus showing leakage from a retinal-retinal anastomosis (white arrowhead). (C) A hot spot consistent with neovascularization is clearly identified by indocyanine green angiography. (D) SD-OCT at baseline revealing elevated PED with subretinal fluid. The visual acuity was 20/40 in the left eye, and the patient was diagnosed as having RAP stage 2 with PED. (E) In total, 6 ranibizumab injections were administered. Finally, at the 67month follow-up examination, the patient's visual acuity had stabilized at 20/40. (F) SD-OCT showing the remaining RPE and outer retina.

Previous studies have reported several prognostic factors in patients with RAP. Chae et al reported that age, sex, and baseline BCVA were prognostic factors for the treatment of RAP. ${ }^{22}$ On the other hand, Cho et al described that baseline BCVA and lesion size were associated with BCVA. ${ }^{18}$ In this study, baseline BCVA and CFT were associated with final BCVA and improvement in BCVA, which was consistent with the findings of previous studies. ${ }^{18,22}$ We speculate that a large CFT could have a large PED, which indicates the progression of RAP. There is a general consensus that more progressive stages show worse visual acuity. Therefore, early detection of RAP and prompt treatment are recommended to maintain visual acuity over the long term.

In our study, factors associated with the development of macular atrophy were also investigated. As a result, age, baseline BCVA, and number of injections were associated with macular atrophy at the final visits. There is a possibility that patients with worse baseline BCVA have already had partial macular atrophy due to disease progression and that the atrophic area expands with longer follow-up. Additionally, older patients may have more oxidative stress-induced degeneration, which could result in an easier progression of macular atrophy. Whether or not the number of injections is associated with the development of macular atrophy is controversial. Grunwald et al reported that monthly injections showed a higher rate of GA than that for PRN. ${ }^{23}$ However, analysis of CATT at 5 years showed that the difference was not statistically significant between the monthly regimen and PRN regimen although the monthly injections showed a higher risk of GA incidence than that of PRN. ${ }^{24}$ Furthermore, the SEVEN-UP study reported that the incidence of macular atrophy was associated with disease activity itself, with 


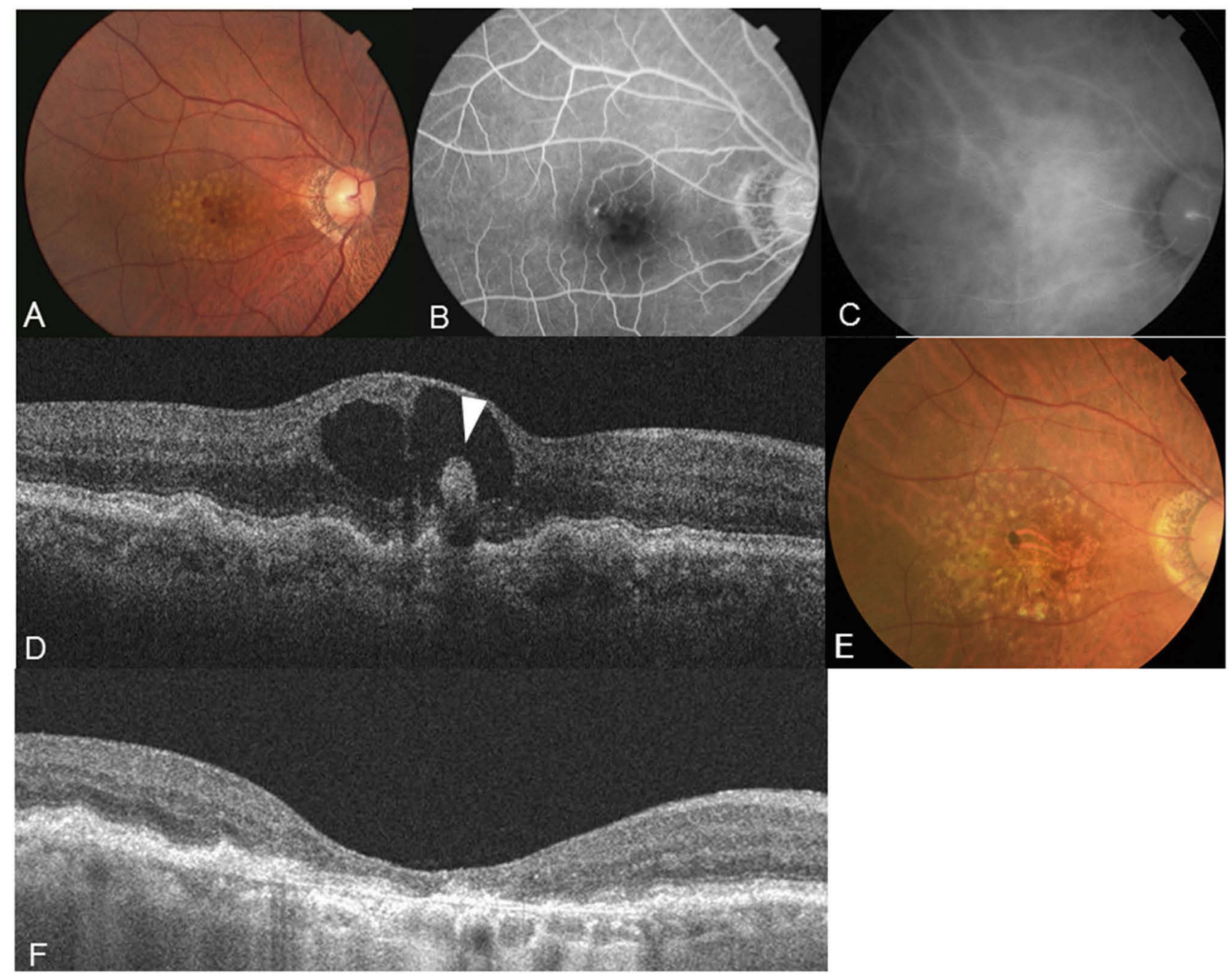

Figure 4 A 78-year-old man presented with decreased visual acuity in his right eye. (A) A color fundus photograph of the left eye showing multiple soft drusen and preretinal hemorrhage. (B) Early-phase fluorescein angiographic image of the fundus showing leakage from a retinal-retinal anastomosis and hypofluorescence consistent with hemorrhage. (C) Indocyanine green angiography showing no abnormal findings. (D) SD-OCT at baseline revealing intraretinal neovascularization without PED (white arrowhead). The visual acuity was 20/50 in the right eye, and the patient was diagnosed as having RAP stage I. (E) In total, 36 ranibizumab injections were administered. Finally, at the 9 -year follow-up examination, the patient's visual acuity had decreased at 20/100. Macular atrophy developed. (F) SD-OCT showing the loss of RPE and outer retina.

leakage for a long time and it causes mechanical damage from ischemic and inflammatory effects. ${ }^{19}$ It is natural that higher disease activity requires a greater number of injections. Although further studies are needed to show an association between anti-VEGF injection itself and macular atrophy, younger age with better visual acuity may be important to prevent the progression of macular atrophy.

A limitation of this study was its retrospective nature. The patients did not all receive the same anti-VEGF agents and the same treatment modality, which may have led to bias. Furthermore, patients who discontinued follow-up were excluded from the study, which also might have influenced the results. The results of this study need to be validated with further prospective studies involving greater uniformity. Lastly, there may be important underlying differences between Japanese and Caucasian patients. Therefore, these results are applicable to a relatively homogenous Asian population, and the same conclusions may or may not be applicable to other patients with similar lesions in the rest of the world.

In conclusion, stabilization of vision in patients with RAP was difficult to achieve by giving intravitreal injections of anti-VEGF agents over the long term regardless of the treatment modality. Earlier detection and treatment at earlier stages are the most important factors for maintaining visual acuity over a long period. 


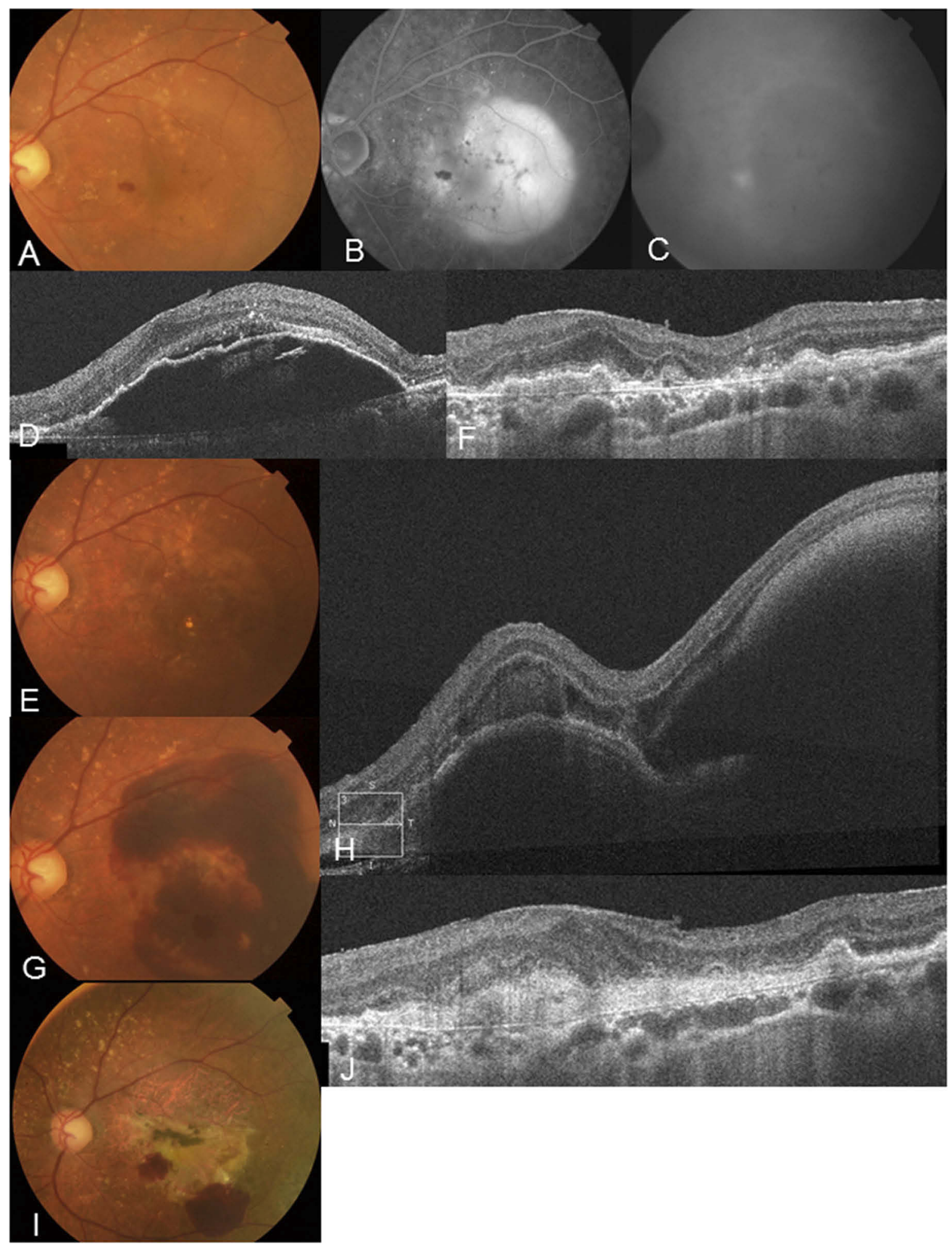

Figure 5 An 84-year-old man presented with metamorphopsia in his left eye. (A) A color fundus photograph of the left eye showing multiple soft drusen and preretinal hemorrhage with PED. (B) Late-phase fluorescein angiographic image of the fundus revealing pooling showing PED and hypofluorescence consistent with hemorrhage. (C) Hot spot consistent with neovascularization is clearly identified by indocyanine green angiography. (D) SD-OCT at baseline revealing elevated PED. The visual acuity was 20/ 25 in the left eye, and the patient was diagnosed as having RAP stage 2 with a PED. (E) After 15 ranibizumab injections were administered, at the 46-month follow-up, PED disappeared and visual acuity improved to 20/16. (F) SD-OCT showing an improvement in the PED. (G) However, 2 days after the 46-month check-up, massive subretinal hemorrhage developed and visual acuity declined to 20/200. (H) SD-OCT showing subretinal hemorrhage and hemorrhagic PED. (I) Finally, after an additional 30 ranibizumab injections, fibrosis with a partial small subretinal hemorrhage was observed at the 108-month follow-up. (J) SD-OCT showing fibrosis with loss of outer retina. Visual acuity decreased to $20 / 1000$. 
However, some complications, such as development of macular atrophy, presence of fibrotic scar, and massive $\mathrm{SRH}$, have resulted in deterioration of visual acuity.

\section{Disclosure}

The authors report no conflicts of interest in this work.

\section{References}

1. Yannuzzi LA, Negrao S, Iida T, et al. Retinal angiomatous proliferation in age-related macular degeneration. Retina. 2001;21:416-434. doi:10.1097/00006982-200110000-00003

2. Viola F, Massacesi A, Orzalesi N, Ratiglia R, Staurenghi G. Retinal angiomatous proliferation: natural history and progression of visual loss. Retina. 2009;29:732-739. doi:10.1097/IAE.0b0 $13 \mathrm{e} 3181 \mathrm{a} 395 \mathrm{cb}$

3. Bottoni F, Massacesi A, Cigada M, et al. Treatment of retinal angiomatous proliferation in age-related macular degeneration: a series of 104 cases of retinal angiomatous proliferation. Arch Ophthalmol. 2005;123:1644-1650. doi:10.1001/archopht.123.12.1644

4. Johnson TM, Glaser BM. Focal laser ablation of retinal angiomatous proliferation. Retina. 2006;26:765-772. doi:10.1097/01.iae.000 0244264.98642.af

5. Boscia F, Furino C, Sborgia L, Reibaldi M, Sborgia C. Photodynamic therapy for retinal angiomatous proliferations and pigment epithelium detachment. Am J Ophthalmol. 2004;138:1077-1079. doi:10.1016/j. ajo.2004.06.072

6. Boscia F, Parodi MB, Furino C, Reibaldi M, Sborgia C. Photodynamic therapy with verteporfin for retinal angiomatous proliferation. Graefes Arch Clin Exp Ophthalmol. 2006;244:1224-1232. doi:10.1007/s00417-005-0205-2

7. Sakimoto S, Gomi F, Sakaguchi H, Tano Y. Recurrent retinal angiomatous proliferation after surgical ablation. Am $\mathrm{J}$ Ophthalmol. 2005;139:917-918. doi:10.1016/j.ajo.2004.10.046

8. Shiragami C, Iida T, Nagayama D, Baba T, Shiraga F. Recurrence after surgical ablation for retinal angiomatous proliferation. Retina. 2007;27:198-203. doi:10.1097/01.iae.0000224938.61915.f0

9. Kuroiwa S, Arai J, Gaun S, Iida T, Yoshimura N. Rapidly progressive scar formation after transpupillary thermotherapy in retinal angiomatous proliferation. Retina. 2003;23:417-420. doi:10.1097/00006982200306000-00027

10. Engelbert M, Zweifel SA, Freund KB. "Treat and extend" dosing of intravitreal antivascular endothelial growth factor therapy for type 3 neovascularization/retinal angiomatous proliferation. Retina. 2009;29:1424-1431. doi:10.1097/IAE.0b013e3181bfbd46

11. Atmani K, Voigt M, Le Tien V, et al. Ranibizumab for retinal angiomatous proliferation in age-related macular degeneration. Eye (Lond). 2010;24:1193-1198. doi:10.1038/eye.2010.9
12. Matsumoto H, Sato T, Morimoto M, et al. Treat-and-extend regimen with aflibercept for retinal angiomatous proliferation. Retina. 2016;36:2282-2289. doi:10.1097/IAE.0000000000001104

13. Inoue M, Arakawa A, Yamane S, Kadonosono K. Long-term results of intravitreal ranibizumab for the treatment of retinal angiomatous proliferation and utility of an advanced RPE analysis performed using spectraldomain optical coherence tomography. Br J Ophthalmol. 2014;98:956960. doi:10.1136/bjophthalmol-2013-304251

14. McBain VA, Kumari R, Townend J, Lois N. Geographic atrophy in retinal angiomatous proliferation. Retina. 2011;31:1043-1052. doi:10.1097/IAE.0b013e3181fe54c7

15. Berg K, Roald AB, Navaratnam J, Bragadottir R. An 8-year follow-up of anti-vascular endothelial growth factor treatment with a treat-and-extend modality for neovascular age-related macular degeneration. Acta Ophthalmol (Copenh). 2017;95:796-802. doi:10.1111/aos.13522

16. Lee JH, Lee MY, Lee WK, Hartnett ME. Incidence and risk factors of massive subretinal hemorrhage in retinal angiomatous proliferation. PLoS One. 2017;12:e0186272. doi:10.1371/journal.pone.0186272

17. Daniel E, Shaffer J, Ying GS, et al. Outcomes in eyes with retinal angiomatous proliferation in the Comparison of Age-Related Macular Degeneration Treatments Trials (CATT). Ophthalmology. 2016; 123:609-616. doi:10.1016/j.ophtha.2015.10.034

18. Cho HJ, Lee TG, Han SY, et al. Long-term visual outcome and prognostic factors of intravitreal anti-vascular endothelial growth factor treatment for retinal angiomatous proliferation. Graefes Arch Clin Exp Ophthalmol. 2016;254:23-30. doi:10.1007/s00417-015-2993-3

19. Bhisitkul RB, Mendes TS, Rofagha S, et al. Macular atrophy progression and 7-year vision outcomes in subjects from the ANCHOR, MARINA, and HORIZON studies: the SEVEN-UP study. Am J Ophthalmol. 2015;159:915-24 e2. doi:10.1016/j.ajo.2015.01.032

20. Baek J, Lee JH, Kim JY, Kim NH, Lee WK. Geographic atrophy and activity of neovascularization in retinal angiomatous proliferation. Invest Ophthalmol Vis Sci. 2016;57:1500-1505. doi:10.1167/iovs.15-18837

21. Jaffe GJ, Martin DF, Toth CA, et al. Macular morphology and visual acuity in the comparison of age-related macular degeneration treatments trials. Ophthalmology. 2013;120:1860-1870. doi:10.1016/j.ophtha.2013.01.073

22. Chae B, Su D, Gal-Or O, Freund KB, Sarraf D. Type 3 neovascularisation: long-term analysis of visual acuity and optical coherence tomography anatomical outcomes. Br J Opthalmol. 2019;103:4348. doi:10.1136/bjophthalmol-2018-311850

23. Grunwald JE, Daniel E, Huang J, et al. Risk of geographic atrophy in the comparison of age-related macular degeneration treatments trials. Ophthalmology. 2014;121:150-161. doi:10.1016/j.ophtha.2013.08.015

24. Grunwald JE, Pistilli M, Daniel E, et al. Incidence and growth of geographic atrophy during 5 years of comparison of age-related macular degeneration treatments trials. Ophthalmology. 2017;124:97-104. doi:10.1016/j.ophtha.2016.09.012
Clinical Ophthalmology

\section{Publish your work in this journal}

Clinical Ophthalmology is an international, peer-reviewed journal covering all subspecialties within ophthalmology. Key topics include: Optometry; Visual science; Pharmacology and drug therapy in eye diseases; Basic Sciences; Primary and Secondary eye care; Patient Safety and Quality of Care Improvements. This journal is indexed on PubMed
Central and CAS, and is the official journal of The Society of Clinical Ophthalmology (SCO). The manuscript management system is completely online and includes a very quick and fair peer-review system, which is all easy to use. Visit http://www.dovepress.com/ testimonials.php to read real quotes from published authors. 